\title{
Evaluación de la política pública para el sector pesquero en el noroeste mexicano: análisis económico y prospectivo
}

\author{
Elizabeth Olmos-Martínez \\ Universidad Autónoma de Baja California Sur, La Paz, México. \\ Email: eliolmos@gmail.com
}

\begin{abstract}
Resumen: El presente estudio se desarrolla en el Noroeste Mexicano en las comunidades de Guaymas, Sonora y Puerto San Carlos, Baja California Sur. Dichas comunidades representan la pesca de sardina en el Proyecto denominado "Unidades Representativas de Producción del Sector Pesquero y Acuícola” desarrollado por SAGARPA y AGROPROSPECTA, tiene como objetivo evaluar políticas públicas gubernamentales en los mercados agroalimentarios de México incluyendo recientemente al sector pesquero. Los datos se obtuvieron por medio de paneles de información donde participaron productores de sardina con datos del año 2008 como base. Se trabajó con el modelo de simulación económica desarrollado para México MexSim, desarrollado en la Universidad de Texas A\&M con indicadores económicos de ingresos y egresos. Los resultados obtenidos muestran que la URP desarrollada en Guaymas proyecta una situación económica general favorable para la actividad, mientras que la URP desarrollada en San Carlos proyecta una situación económica general moderada.
\end{abstract}

Palabras clave: política, sardina, proyección, análisis económico.

\section{Evaluation of public policy for the fishing industry in northwest Mexico: economic analysis and prospective}

\begin{abstract}
This study was developed in the northwest Mexican communities of Guaymas, Sonora and Puerto San Carlos, Baja California Sur. These communities represent the sardine fishery in the project entitled "Production Representative Unit of the Fisheries and Aquaculture" developed by SAGARPA and AGROPROSPECTA, which aims to assess government policies in food markets of Mexico including most recently the fisheries sector. Data were obtained through information panels where sardine producers participated with 2008 data as base. Worked with the economic simulation model developed for Mexico MexSim, developed at the University of Texas A \& M with economic indicators of income and expenses. The results show that the URP developed in Guaymas projects a favorable overall economic activity, while the URP developed in San Carlos projects a moderate general economic situation.
\end{abstract}

Key words: politics, sardine, projection, economic analysis. 


\section{Avaliação de políticas públicas para a indústria de pesca no noroeste do México: análise económica e prospectivo}

Resumo: Este estudo foi desenvolvido nas comunidades mexicanas noroeste de Guaymas, Sonora e Puerto San Carlos, Baja California Sur. Essas comunidades representam a pesca da sardinha no projeto intitulado "Unidade de Produção Representante das Pescas e Aquicultura”, desenvolvido pela SAGARPA e AGROPROSPECTA, tem como objetivo avaliar as políticas governamentais nos mercados de alimentos do México, incluindo mais recentemente o sector das pescas. Os dados foram obtidos através de painéis de informação onde os produtores de sardinha participaram com dados de 2008 como base. Trabalhou-se com o modelo de simulação econômica desenvolvida para o México MexSim, desenvolvido na Universidade do Texas A \& M, com indicadores econômicos de renda e despesas. Os resultados mostram que a URP desenvolvida em Guaymas projeta uma atividade econômica global favorável, enquanto a URP desenvolvida em San Carlos projeta uma situação geral económica moderada.

Palavras-chave: política, sardinha, projeção, análise econômico.

$* * *$

\section{Introducción}

La pesca en México tiene un valor económico, social y alimentario con fuertes impactos regionales en la que su elevado potencial de producción ha contribuido en la solución de problemas alimentarios y de generación de empleos. Esta actividad se realiza en tres áreas geográficas principales: 1) litoral del Pacífico; 2) litoral del Golfo y El Caribe; y 3) aguas continentales (Juárez, et al, 2007).

Cifras estimadas de 2006 muestran que en el litoral del Pacífico se realizó la captura del 79\% del volumen de producción pesquera; en el litoral del Golfo y el Caribe se concentró alrededor del 19\% del volumen y sólo 2\% en aguas continentales. En 2006, cifras estimadas de la Comisión Nacional de Acuacultura y Pesca (CONAPESCA) muestran que tres entidades federativas producen cerca del 60\% del volumen de pesca (Baja California Sur 10\%, Sonora 35\% y Sinaloa 14\%) equivalente al $47 \%$ del valor total de la producción del sector (Ibid).

Baja California Sur (BCS) cuenta con un litoral de 2,575.39 km (22\% respecto al total mexicano), de los cuales $1,400 \mathrm{~km}$ corresponden a la costa occidental y $1 \mathrm{~m} 305 \mathrm{~km}$ a la costa oriental (Ojeda-Ruiz de la Peña y Sánchez-Hernández 2003).

De acuerdo con las características anteriores BCS es el estado mexicano con mayor litoral del país y con un enorme potencial pesquero. En las costas de BCS, se han identificado 650 especies que pueden ser utilizadas para consumo humano e industrialización, actualmente se explotan 122 grupos de especies comestibles entre las que destacan, por su volumen de captura, la pesquería de sardina (Sardinops sagax), túnidos y almejas 
(Thunnus thynnus y megapitaria squalida), por otro lado se cuenta con recursos de alto valor económico como las pesquerías de abulón (Haliotis corrugata), Langosta roja (Panulirus interruptus) y Camarón café y azul (Farfantepenaeus aztecus), (Penaeus stylirostris), las cuales han sido el eje de desarrollo social en algunas comunidades pesqueras de BCS, en particular la zona pacífico norte (Ojeda-Ruiz de la Peña y Sánchez-Hernández 2003).

Según Quiñones, et al (2009), para aprovechar el recurso pesquero es necesario establecer formas de captura distintas a las actualmente utilizadas en el estado, estas deben ser embarcaciones de altura que tengan autonomía por varios días, con capacidad de conservación a bordo y motores diesel. Además, mencionan que existen acciones que pueden mejorar la calidad de los productos, la diversificación de sus formas de presentación y a la incidencia en mercados que ofrecen mejores precios, tal es el caso de las especies como lenguados, caracol panocha, tiburón, atún y sardina.

Existen algunas limitantes detectadas para el desarrollo de la pesquería en BCS, algunas de ellas son: a) un deficiente manejo de los productos pesqueros, desde su extracción hasta su transportación a los centros de consumo, b) excesivo intermediarismo y acaparamiento, lo anterior provoca que la comercialización sea deficiente con un limitado beneficio para los productores; c) además se ha identificado que se explotan los recursos bentónicos hasta sus límites cercanos al agotamiento (Casas y Ponce, 1996).

En México, el sector primario es de vital importancia para el gobierno; no obstante, los esfuerzos para impulsar este sector, han sido insuficientes para estimular el desarrollo de éste. Tal es el caso del sector pesquero. A pesar de contar con las condiciones territoriales, la industria pesquera es considerada como una de las actividades más complejas del sector primario debido a factores que van desde las condiciones ambientales, hasta las económicas, políticas y sociales.

Es por eso que la Dirección General de Estudios Agropecuarios y Pesqueros de la Subsecretaria de Fomento a los Agronegocios de la Secretaría de Agricultura, Ganadería, Pesca y Alimentación (SAGARPA), que a través del componente Desarrollo de Mercados y Planeación Prospectiva del Programa de Soporte, aportó financiamiento necesario para la realización del "Análisis y Prospectiva una herramienta para la toma de decisiones de políticas públicas" por medio de recursos administrados por la Universidad Autónoma de Chapingo en su calidad de Agente Técnico del Proyecto y de la Red Mexicana de Investigación en Política Agroalimentaria (AGROPROSPECTA), que tiene como principal objetivo generar análisis económico de política pública de manera objetiva y oportuna respecto a los mercados agroalimentarios de México usando como herramienta modelos de simulación. Actualmente AGROPROSPECTA está integrada por investigadores y académicos especializados en temas relacionados con la economía del sector agroalimentario de México. 


\section{Marco teórico}

La historia de la modelación de simulación Monte Carlo data desde 1965 cuando Halter y Dean en 1965 crearon modelos de riesgo para granjas, no obstante fue hasta 1981 cuando Richardson y Nixon crearon el modelo llamado FLIPSIM (Firm Level Policy Simulation) teniendo un avance rápido hasta la actualidad (Richardson y Nixon, 1986). Excel y el análisis de riesgo se complementan para hacer más fácil construir modelos basados en riesgo, por lo que se desarrollo SIMETAR, que es el más nuevo y más adaptable modelo para análisis de riesgo (Taller MEXSIM-SIMETAR).

SIMETAR fue desarrollado en Centro de Política Agroalimentaria de la Texas A\&M University (TAMU) durante 1999-2005. Es usado para análisis de riesgo basado en políticas de granjas y sectores en Estados Unidos; fue comercializado con la licencia de TAMU en 2005.

Con el paso del tiempo un gran número de cuestionamientos relacionados con los pastizales y uso de recursos naturales han sido analizados, sin embargo, muchos de estos análisis no han logrado proporcionar la fiabilidad, robustez y utilidad para los gestores estadounidenses y tomadores de decisiones políticos de que los esfuerzos dirigidos a la producción agronómica ha producido (Bernardo y Conner, 1990). Muchos factores han contribuido a la dificultad analítica, incluyendo la complejidad del sistema ya que se trata de productos que provienen de recursos vivos. Conner (1994) sugiere que aminorar los impactos socioeconómicos de cambios en los pastizales y recursos naturales podría lograrse mediante un procedimiento mediante el cual una región se caracteriza por el modelado de un conjunto de fincas o granjas representativas usando la salida del modelo ce crecimiento de la planta como entrada a un nivel de empresa modelo de desempeño económico.

Desde los inicios de FLIPSIM, Richardson y Dixon (1986) desarrollaron dicho modelo y se caracteriza por ser dinámico, recursivo y estocástico, y, simula los impactos en las características financieras y económicas de una granja o rancho específico como alternativa institucional, técnica o gerencial del desarrollo de sus propios periodos específicos (Souza Neto, et al., 2001). Este modelo es oportuno para iniciar un modelo integrado que muestra la composición de especies (plantas y animales) que pueden ser integradas dentro de la evaluación de impactos socioeconómicos, ambientales o de otros cambios (Ibid). El modelo se ejecuta de modo estocástico, y en consecuencia, prevé resultados para analizar estrategias alternativas de gestión y determinar los efectos sobre la viabilidad económica de la empresa representante en escenarios alternativos (Souza Neto, et al., 1996).

FLIPSIM simula precio y riesgo para el rendimiento, las actividades económicas anuales de una granja representativa sobre periodos de múltiples años de planificación (Lien, 2003). El modelo utiliza ecuaciones que representan las distribuciones de probabilidad. Estas han sido usadas 
para análisis de políticas (Knutson, et al., 1997), comparación estrategias de gestión de riesgo (Knutson, et al., 1998), evaluación tecnológica (Nyangito, et al., 1996), análisis financiero (Hughes, et al., 1985), etc.

En general, muchos han sido los estudios de granjas representativas de producción agropecuaria que han utilizado el modelo FLIPSIM en Estados Unidos, que más tarde se convirtió en el modelo SIMETAR, y la versión para México, desarrollado en TAMU, se le conoce con el nombre de MEXSIM. La experiencia para México se ha desarrollado en la Secretaría de Agricultura, Ganadería, Pesca y Alimentación (SAGARPA) con el apoyo del Agente técnico de la Red AGROPROSPECTA.

En 2008, AGROPROSPECTA desarrollo 60 Unidades Representativas de Producción (URP) de diez productos agrícolas y pecuarios (AGROPROSPECTA, 2009), en 2009 sumaron 180 URP de productos agrícolas, pecuarios y pesqueros. En el caso de las pesquerías los productos estudiados incluyen, sardina, abulón-langosta, calamar y camarón para la pesca y camarón y tilapia para acuicultura.

El propósito es presentar la proyección de la viabilidad económica y financiera de estas URP para el periodo 2009-2018, tomando como base el comportamiento económico de las mismas en 2008. El análisis de las URP utiliza proyecciones macroeconómicas como precios, tasas de inflación y variables de política pública.

Los resultados intentan describir como podrían ser las tendencias sectoriales bajo un esquema de persistencia de las condiciones económicas y de política pública actual.

Sirve como herramienta para la evaluación prospectiva del impacto de los cambios a la política pública y situaciones coyunturales al sector. Genera las proyecciones base del sector primario en el largo plazo. Estas indican las tendencias del sector si la perspectiva del entorno macroeconómico y de política se mantiene constante.

El modelo tiene como propósito ser una herramienta técnica para el análisis, la planeación y la toma de decisiones en el sector agropecuario y pesquero de México. El enfoque ayuda a formular recomendaciones de utilidad práctica para la toma de decisiones significativas y oportunas en materia de planeación de la política pública en base a los resultados de gestión (Callejas, 2008).

La evaluación externa permite integrar una base de conocimiento útil y práctico para aspectos como lo son establecer una nueva práctica de evaluación de los programas sectoriales destinados al sector agropecuario y pesquero, implementar un sistema de seguimiento continuo de los impactos y gestión de los programas de apoyo, proporcionar un modelo de diseño y cálculo de indicadores de desempeño, apoyar la creación y validación de bases de datos e información para la evaluación de programas agropecuarios 
y pesqueros, difundir la información derivada de los procesos de evaluación a los actores y beneficiarios de los apoyos, de manera oportuna y con altos niveles de confiabilidad, mejorar la base técnica para la recolección y análisis de datos y aportar recomendaciones de política pública para la planeación de la política sectorial (Ibid).

\section{Metodología}

El análisis microeconómico de simulación se llevó a cabo en el modelo econométrico de simulación agroeconómica MEXSIM desarrollado en el centro de Política Agroalimentaria de la Texas A\&M University bajo los siguientes indicadores: situación económica general (reservas finales de efectivo, capital neto real), ingresos en efectivo (ventas, transferencias, otros ingresos), ingreso neto en efectivo y reservas en efectivo; además de un balance general (ingresos - costos). Los resultados ofrecen información sobre la viabilidad económica de las URP bajo las condiciones productivas, tecnológicas y económicas observadas al momento de realizar el panel en el año base 2008.

Los datos para el estudio son recabados por medio de la técnica de paneles de trabajo dirigidos a un grupo de productores. Los paneles se conforman por grupos de productores expertos de cada región y que caracterizan un sistema de producción, un nivel tecnológico y una escala dentro de un rango de capacidad productiva. En esos grupos participan preferentemente entre cinco y diez productores, cuyas unidades de producción particulares tienen características similares, dicho panel es convocado por un facilitador, conocedor de la actividad realizada en la región con cercanía y capacidad de convocatoria de los productores.

Utilizando el proceso de consenso, en cada panel se recaba información relacionada con ingresos y costos de producción, así como parámetros técnicos y sistemas de comercialización para un año base, por lo que la escala, el sistema de producción, nivel tecnológico, precio de compra de los insumos y venta de los productos, para un año en particular (año base), son acordados por consenso por los panelistas para conformar una URP.

Se desarrollaron dos URP típicas y representativas del sector pesquero en el noroeste mexicano para el producto sardina (Sardinops sagax), una de las URP se desarrolló en Guaymas, Sonora y la otra en Puerto San Carlos, Baja California Sur.

Una URP, es aquella unidad de producción que sin representar a un productor en particular, tipifica virtualmente las actividades y decisiones de los productores participantes en un panel, a la vez la URP representa una unidad de producción característica de una escala y un sistema de producción particular de una región productora del país.

La denominación de las URP se realizó de la siguiente manera: las 
dos primeras letras representan el nombre del estado de la república donde se encuentran localizadas, las siguientes dos letras representan la pesquería, y finalmente el número representa el tamaño de la escala de producción.

\section{Caracterización de las URP representativas:}

SOSD60000: URP ubicada en Guaymas, Sonora, cuenta con 4 barcos propios, cada uno tiene una capacidad de 200 toneladas de almacenamiento, el motor de las embarcaciones es de $600 \mathrm{hp}$, todos los barcos cuentan con equipo de refrigeración, cuentan con redes de de 320 brazas, de 40 de longitud por 40 de caída, sistema hidráulico (pasteca) y equipo electrónico (sonar, radar, navegador por satélite, video sonda). La mayor parte de la producción es destina a la reducción y comercializada localmente.

BCSSD12000: URP ubicada en Puerto San Carlos, Baja California Sur, cuenta con 1 barco propio, con capacidad de 120 toneladas de almacenamiento, el motor de la embarcación es de $700 \mathrm{hp}$, cuenta con equipo de refrigeración, cuenta con redes de 270 brazas, sistema hidráulico (pasteca) y equipo electrónico (sonar, radar, navegador por satélite, video sonda). La mayor parte de la producción se destina al consumo humano (enlatado, congelado y fresco), es comercializada localmente.

Con el fin de asegurar la validez y representatividad de los datos obtenidos, los resultados del año base son validados por los panelistas. La validación se realiza en un proceso similar al de censo de paneles, donde se convoca nuevamente a los participantes del panel original, con la intención de verificar que la información sea completa y correcta, además de comprobar que la situación económica de la URP refleje la situación para el año base. Del mismo modo se verifica que los panelistas estén de acuerdo en que el análisis prospectivo refleje adecuadamente sus expectativas sobre el sector.

La siguiente etapa es el análisis prospectivo y estocástico. Este tipo de análisis desarrolla proyecciones de las principales variables económicas a través de un horizonte de planeación (diez años) tales como ingresos y costos del año base. Con el fin de que se simule el riesgo enfrentado por la actividad, las proyecciones se realizan considerando los precios y rendimientos históricos observados por los productores a nivel nacional. Para tener aún mayor representatividad, se realizan ajustes que recogen el nivel local de precios para los diferentes insumos en cada región productiva incluida en el análisis.

El modelo MEXSIM utilizado, captura las variables obtenidas a través de los paneles y permite realizar proyecciones estocásticas de los principales indicadores a nivel unidad de producción. Los resultados obtenidos ofrecen información sobre viabilidad económica de la URP bajo las condiciones productivas, tecnológicas y económicas observadas al momento de realizar el panel (Ricardson y Nixson, 1986). 
Las proyecciones empleadas en el análisis incluyen variables como producto interno bruto, tipo de cambio promedio, precio del petróleo, índice nacional de precios del consumidor y tasas de interés nominal.

Con el fin de que los resultados muestren el riesgo enfrentado por los productores, se incluyeron precios y rendimientos históricos, permitiendo estimar la probabilidad de obtener reservas finales de efectivo negativas y la probabilidad de enfrentar pérdidas de capital neto real, dentro de los indicadores de viabilidad económica de las URP (Callejas, 2008).

\section{Resultados}

En el año base de 2008, las características generales de las URP muestran que la URP denominada BSSD12000 es la de menor escala con una capacidad total del 12,000 toneladas de pesca anual, con un valor de los activos equivalente a $\$ 13,305,000$ (pesos mexicanos) y con ingresos totales de $\$ 10$, 992,000 (pesos mexicanos), de los cuales el $100 \%$ son por concepto de la venta de sardina. En el caso de la URP denominada SOSD60000 es la de mayor escala con una capacidad total de 60,000 toneladas de pesca anual, cuenta con un valor de los activos de \$96,350,000 (pesos mexicanos) y con ingresos totales de $\$ 48,200,000$ (pesos mexicanos), de los cuales el 100\% son por concepto de la venta de sardina (cuadro 1).

\section{Cuadro 1. Características de las URP}

\begin{tabular}{cccccc}
\hline & $\begin{array}{c}\text { Capacidad total } \\
\text { (ton) }\end{array}$ & $\begin{array}{c}\text { Valor de los } \\
\text { activos } \\
(\$ 1,000)\end{array}$ & $\begin{array}{c}\text { Ingresos totales } \\
(\$ 1,000)\end{array}$ & $\begin{array}{c}\text { Capacidad de } \\
\text { captura } \\
\text { (ton) }\end{array}$ & $\begin{array}{c}\text { Ingreso pesca/ } \\
\text { ingreso total } \\
(\%)\end{array}$ \\
\hline BSSD12000 & 12,000 & 13,305 & 10,992 & 12,000 & 100 \\
SOSD60000 & 60,000 & 96,350 & 48,200 & 60,000 & 100 \\
\hline
\end{tabular}

La situación económica general durante el periodo 2009-2018 resume la eficiencia económica, liquidez y solvencia de la URP y se despliega en lo que se conoce como gráfica de semáforo, la cual muestra los datos en celdas de colores; si la situación es favorable, la celda se muestra de color gris claro, si la situación es moderada, se muestra de color gris oscuro y si la situación es desfavorable, la celda se muestra de color negro. Dicha gráfica arroja la situación general, las probabilidades de reservas finales de efectivo negativas y la descapitalización, todo esto en términos de probabilidad y representadas en porcentaje.

Una URP es considerada en situación económica favorable cuando el promedio de las probabilidades de obtener reservas finales de efectivo negativas y de perder capital (patrimonio o capital neto real) es menor o igual a $25 \%$ (gris claro). Si la probabilidad de este evento es superior a 
25\% y menor o igual a 50\%, la situación financiera de la URP es considerada moderada (gris oscuro). Cuando es superior a 50\%, la URP es considerada en situación económica desfavorable (negro).

Para el año 2009, la URP SOSD60000 presenta una situación general favorable (gris claro), mientras la otra una situación moderada (gris oscuro), para el año 2018, la situación económica se mantiene en ambas URP. La URP SOSD60000 presenta 18 y 5\% de probabilidad de obtener las reservas finales de efectivo negativas, en los años 2008 y 2018 respectivamente, la cual se considera baja probabilidad (gris claro), en el mismo sentido, la URP BSSD12000 presenta 44 y 40\% de probabilidad, lo que se considera media (gris oscuro). La probabilidad de descapitalización de la URP SOSD60000 es de 19 y 4\%, para 2008 y 2018 respectivamente, considerada baja (gris claro), en el caso de la URP BSSD12000 la probabilidad es de 56 y 43\%, para 2008 y 2018 respectivamente, lo que se considera media (gris oscuro) (cuadro 2).

\section{Cuadro 2. Situación económica durante el periodo 2009-2018 (\%)}

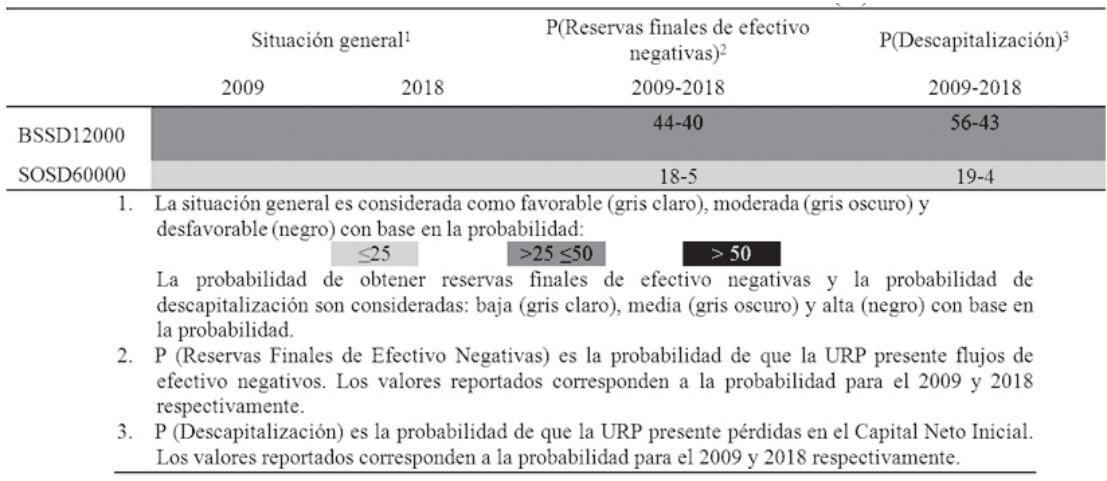

Para el año 2008, la estructura de ingresos y egresos muestra la siguiente distribución para la URP SOSD60000: a) Ingresos, 93\% venta de sardina y $7 \%$ trasferencias y/o subsidios; b) Egresos, combustible 29\%, mantenimiento $21 \%$, mano de obra $29 \%$, indirectos $8 \%$ y otros $20 \%$ (gráficas 1 y 2). El alto costo de la mano de obra se debe a que incluye la tripulación de 4 barcos con un total de 40 empleados, además de 80 empleados temporales para el mantenimiento de las redes. El gasto de mantenimiento del barco incluye conceptos como, astillero, maquinaria, refrigeración, equipo eléctrico, carpintería y equipo de pesca; cabe mencionar que el mantenimiento del barco genera un costo alto debido a que son modelos "viejos" (más de 30 y 40 años), los cuales constantemente necesitan de mantenimiento mayor. 


\section{Gráfica 1. Estructura de ingresos de SOSD60000, año base 2008}

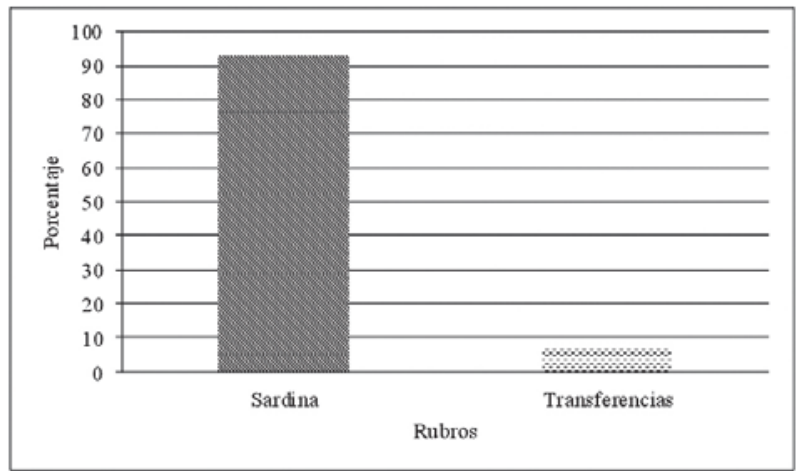

Fuente: Elaboración propia en base a datos del panel.

\section{Gráfica 2. Estructura de egresos de SOSD60000, año base 2008}

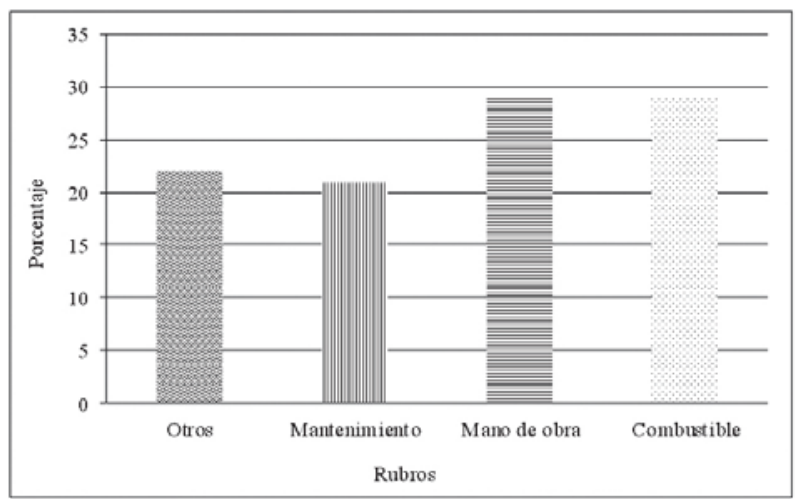

Fuente: Elaboración propia en base a datos del panel.

Para la URP BSSD12000 la estructura de ingresos y egresos fue la siguiente: a) Ingresos, venta de sardina monterrey $73 \%$, venta de sardina bocona $16 \%$, trasferencias y/o subsidios 5\%, otros ingresos 7\%; b) Egresos, mantenimiento $27 \%$, mano de obra $26 \%$, combustible $23 \%$ y otros $24 \%$ (Gráficas 3 y 4). En este caso el gasto en mano de obra incluye 16 personas en tripulación del barco y 24 trabajadores de tiempo parcial quienes hacen el trabajo de rederos y carpinteros en la temporada alta (marzo-octubre). El alto costo del mantenimiento del barco y equipos de pesca también saltan a la vista con el $27 \%$ de los egresos, en este caso el barco cuenta con una antigüedad de 20 años. 
Gráfica 3. Estructura de ingresos de BSSD12000, año base 2008

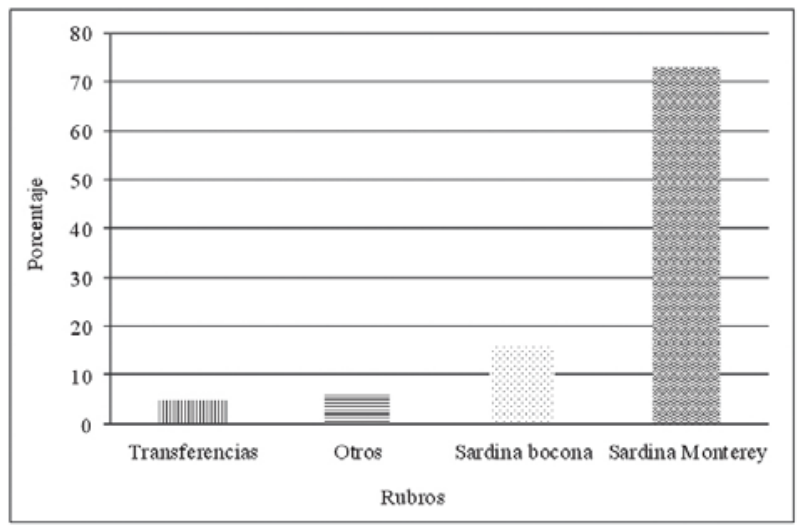

Fuente: Elaboración propia en base a datos del panel.

\section{Gráfica 4. Estructura de egresos de BSSD12000, año base 2008}

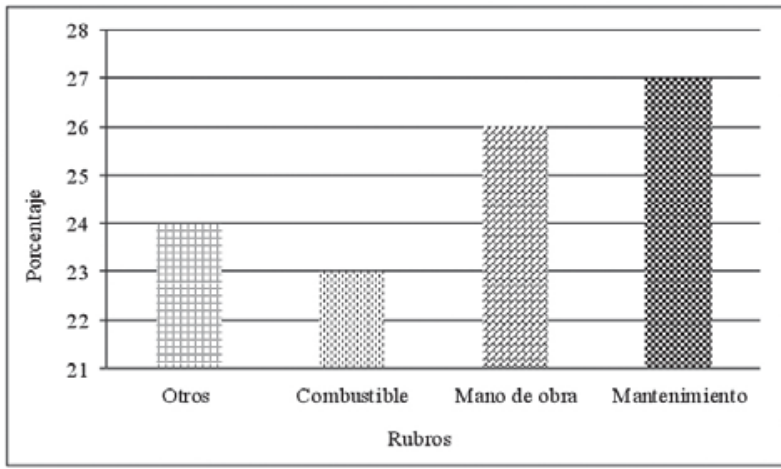

Fuente: Elaboración propia en base a datos del panel.

El cuadro 3 muestra los parámetros económicos proyectados para la URP SOSD60000, en los que se puede distinguir que el ingreso neto para el año 2008 asciende a \$14,300,000 (pesos mexicanos) con un incremento del $96 \%$ para el año 2018, además se observa que las probabilidades de descapitalización y reservas finales negativas están por abajo del $20 \%$ en todo el periodo, lo que muestra una proyección favorable para esta URP. En general, para la URP SOSD60000 se observa una tendencia favorable para los ingresos netos en efectivo ya que ninguna de sus probabilidades tiene números negativos durante el periodo 2009-2018. 


\section{Cuadro 3. Parámetros económicos proyectados, SOSD60000 (miles de pesos)}

\begin{tabular}{|c|c|c|c|c|c|c|c|c|c|c|c|}
\hline & 2008 & 2009 & 2010 & 2011 & 2012 & 2013 & 2014 & 2015 & 2016 & 2017 & 2018 \\
\hline $\begin{array}{l}\text { Ingreso } \\
\text { pesquero }\end{array}$ & 45,000 & 49,581 & 52,839 & 55,529 & 60,209 & 59,797 & 57,626 & 65,406 & 68,834 & 68,851 & 72,605 \\
\hline Transferencias & 3,200 & 3,200 & 3,200 & 3,200 & 3,200 & 3,200 & 3,200 & 3,200 & 3,200 & 3,200 & 3,200 \\
\hline Ingreso total & 48,200 & 52,781 & 56,039 & 58,729 & 63,409 & 62,997 & 60,826 & 68,606 & 72,034 & 72,051 & 75,805 \\
\hline Gasto total & 33,900 & 35,329 & 36,812 & 38,090 & 39,369 & 40,641 & 41,976 & 43,326 & 44,826 & 46,333 & 47,756 \\
\hline Ingreso neto & 14,300 & 17,452 & 19,228 & 20,640 & 24,040 & 22,335 & 18,851 & 25,280 & 27,209 & 25,718 & 28,049 \\
\hline $\begin{array}{l}\text { Reservas } \\
\text { Finales de } \\
\text { efectivo }\end{array}$ & 15,270 & 27,158 & 40,493 & 55,091 & 72,805 & 89,115 & 102,335 & 121,069 & 142,015 & 161,758 & 183,793 \\
\hline $\mathrm{P}(\mathrm{DFE})(\%)^{1}$ & & 18 & 13 & 13 & 4 & 12 & 18 & 9 & 6 & 9 & 5 \\
\hline $\mathrm{P}($ Desc. $)(\%)^{2}$ & & 19 & 29 & 18 & 17 & 13 & 15 & 11 & 14 & 11 & 4 \\
\hline
\end{tabular}

1 P DFE): Probabilidad de Déficit de flujo de efectivo.

2 P(Desc.): Probabilidad de Descapitalización.

Fuente: Resultados de MEXSIM, SOSD60000

El cuadro 4 muestra los parámetros económicos proyectados para la URP BSSD12000, donde se observa que el ingreso neto para el año 2008 asciende a $\$ 1.005 .000$ (pesos mexicanos) el cual se proyecta favorable para el año 2018 con incremento de 324\%, en el caso de las probabilidades descapitalización y reservas finales negativas se observan por abajo del $60 \%$ y 50\%, respectivamente, lo que indica una proyección desfavorable para la URP.

\section{Cuadro 4. Parámetros económicos proyectados, BSSD12000 (miles de pesos)}

\begin{tabular}{|c|c|c|c|c|c|c|c|c|c|c|c|}
\hline & 2008 & 2009 & 2010 & 2011 & 2012 & 2013 & 2014 & 2015 & 2016 & 2017 & 2018 \\
\hline $\begin{array}{l}\text { Ingreso } \\
\text { pesquero }\end{array}$ & 9,690 & 10,7160 & 11,296 & 12,411 & 13,039 & 12,658 & 12,443 & 13,313 & 14,592 & 14,1679 & 16,054 \\
\hline Transferencias & 560 & 560 & 560 & 560 & 560 & 560 & 560 & 560 & 560 & 560 & 560 \\
\hline Ingreso total & 10,992 & 12,054 & 12,674 & 13,820 & 14,484 & 14,138 & 13,962 & 14,870 & 16,201 & 16,339 & 17,756 \\
\hline Gasto total & 9,987 & 10,433 & 10,964 & 11,374 & 11,780 & 12,176 & 12,606 & 13,054 & 13,554 & 14,051 & 14,498 \\
\hline Ingreso neto & 1,005 & 1,620 & 1,709 & 2,446 & 2,707 & 1,962 & 1,357 & 1,817 & 2,647 & 2,289 & 3,258 \\
\hline $\begin{array}{l}\text { Reservas } \\
\text { Finales de } \\
\text { efectivo }\end{array}$ & 434 & 1,425 & 2,473 & 4,233 & 6,225 & 7,458 & 8,062 & 9,103 & 10,942 & 12,403 & 14,816 \\
\hline $\mathrm{P}(\mathrm{DFE})(\%)^{1}$ & & 44 & 49 & 43 & 41 & 48 & 55 & 50 & 45 & 46 & 40 \\
\hline $\mathrm{P}\left(\right.$ Desc.) $(\%)^{2}$ & & 56 & 56 & 48 & 46 & 49 & 54 & 52 & 59 & 57 & 43 \\
\hline
\end{tabular}

1 P(DFE): Probabilidad de Déficit de flujo de efectivo.

2 P(Desc.): Probabilidad de Descapitalización.

Fuente: Resultados de MEXSIM, BSSD12000 
Las gráficas 5 y 6 muestran el ingreso total, gastos totales e ingresos netos en efectivo para el periodo 2009-2018, se observa que en la URP SOSD60000 los ingresos totales superan al total de los gastos lo que genera una rentabilidad sostenida de la URP durante el periodo, con altas probabilidades de mantenerse en el tiempo y con ingresos netos en efectivo positivos. En el caso de la URP BSSD12000 los ingresos netos en efectivo son muy bajos, pero positivos, ya que los gastos de la URP son muy altos apenas menores a los ingresos totales, lo que genera un sentido de alerta, aunque se percibe que durante el periodo de 2009-2018 existen años en los que el ingreso neto en efectivo se encuentra por abajo del año 2009 llegando a su máximo en el año 2018, es decir la URP puede mantenerse en el tiempo con las condiciones actuales ya sean tecnológicas, económicas y/o de subsidios del gobierno.

\section{Gráfica 5. Ingresos totales, gastos totales e ingresos netos de la URP SOSD60000, periodo 2009-2018.}

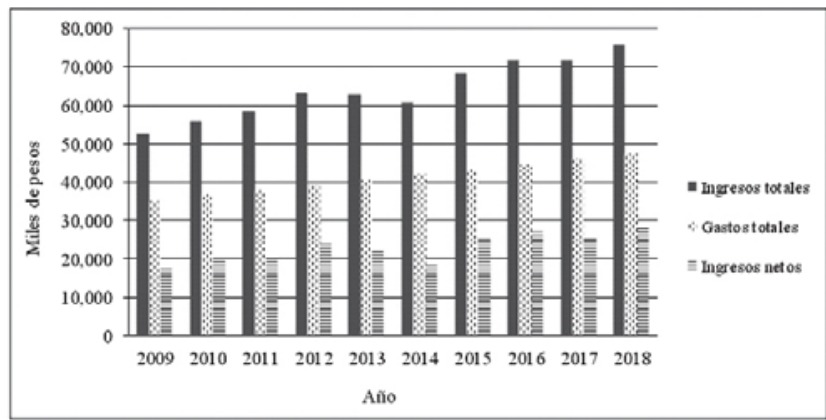

Fuente: Elaboración propia en base a los resultados de MEXSIM, SOSD60000.

Gráfica 6. Ingresos totales, gastos totales e ingresos netos de la URP BSSD12000, periodo 2009-2018.

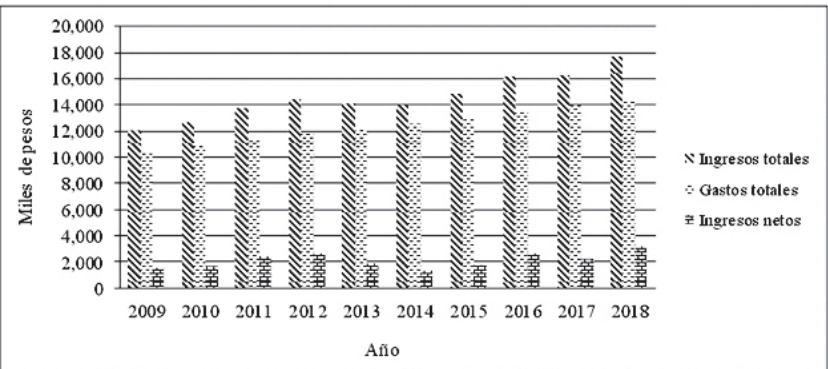

Fuente: Elaboración propia en base a los resultados de MEXSIM, BSSD12000. 
Los panelistas de la URP ubicada en Guaymas, Son., SOSD60000 manifestaron algunas características operativas de la actividad pesquera entre las cuales podemos rescatar la falta de oficinas y taller de mantenimiento, así como almacén de resguardo de bienes ya que generalmente las instalaciones físicas son de la reductora, la cual renta al barco un taller de flora, reparación de redes, tibores, tanque para diesel marino, etc., por lo que se genera un costo de renta. Además se manifestó la falta de un muelle propio para no erogar presupuesto a la Administración Portuaria Integral.

Asimismo los permisos de pesca que tienen una vigencia de 2 a 4 años con un costo de \$35,000 (pesos mexicanos) anuales por embarcación, los panelistas sugieren que requieren las concesiones de los muelles para reducir costos y hacer eficiente el manejo. Sobre obtención de créditos, manifestaron que los obtienen por parte de los proveedores, sin intereses y aun mes, con el compromiso de venta del producto.

El panel manifestó tener el problema de flota de barcos antiguos, los cuales requieren mantenimiento mayor cada temporada, además el mantenimiento preventivo mensual. Se requiere renovar la flota pesquera con créditos especiales con intereses bajos. Asimismo comentaron que la vida promedio de los barcos es de 25 años y que ellos están trabajando con embarcaciones más antiguas y les invierten grandes cantidades de dinero en mantenimiento.

Dentro de la capacitación para la operación de los barcos tuvieron acceso a cursos de capacitación para la tripulación a través del Fideicomisos Instituidos en Relación a la Agricultura (FIRA). Manifestaron que lo que les interesa es capturar el mejor producto con mayores proteínas y posicionarse del mercado extranjero.

Por su parte los panelistas de Puerto San Carlos manifestaron que prácticamente la operación de la embarcación es de subsistencia y que as posibles soluciones son políticas públicas dirigidas a fortalecer el mercadeo, precios y destino del producto. Además dijeron ser afectados por fenómenos meteorológicos como ciclones y años niño y niña. Sugieren apoyos gubernamentales dirigidos al sector que sean paliativos a la problemática existente.

\section{Conclusiones y recomendaciones}

En México, el 68\% de la captura de sardina se desarrolla en los estados de Baja California Sur y Sonora (SAGARPA, 2007). La sardina es un producto pesquero que se destina para el consumo humano y para reducción (harina de pescado), en el noroeste mexicano es un producto de gran importancia para la actividad pesquera.

En el caso de las URP que representan la actividad pesquera de sardina en Guaymas y en Puerto San Carlos, bajo las condiciones analizadas 
en 2008 (económicas, tecnológicas y de política pública), la actividad de pesca de sardina en el noroeste mexicano es rentable.

Para la URP desarrollada en Guaymas, Sonora (SOSD60000) un análisis costo beneficio da como resultado 2.37, lo que indica que por cada peso invertido se obtuvo un beneficio del $137 \%$ para el año 2008 , se observa en las proyecciones a diez años que este indicador caerá hasta tener un rendimiento de $70 \%$ para el 2018. El análisis costo-beneficio para la URP BSSD12000 resulta de 1.10, lo que indica se obtendrá un beneficio del $10 \%$ para 2008, en ese sentido la proyección para el 2018 muestra un beneficio de $22 \%$.

El ingreso para los próximos años en la URP BSSD12000 presenta una tendencia positiva en la mayoría de los años, respecto al año anterior, sustentada en los rendimientos y el precio del producto, hay que recordar que el esfuerzo proyectado fue constante a partir del año base, sin considerar factores ambientales y de cambio de tecnología.

El ingreso para la URP SOSD60000 muestra una tendencia positiva, presentando un decremento en el año 2014 y retomando el incremento para el 2018. Dado que la actividad pesquera es una actividad económica con riesgo, es importante la planificación para aminorarlo.

Se recomienda que SAGARPA continúe con la evaluación de las políticas públicas del sector pesquero lo cual permitirá conocer el panorama actual y prospectivo del desarrollo de la actividad. Además es necesario implementar un sistema de seguimiento continuo de los impactos y de la gestión de los programas de apoyo presentados en estos modelos como "transferencias”. Además, es necesario difundir la información derivada de los procesos de evaluación para que los actores continúen aportando su conocimiento e información necesaria para la modelación.

Son varias las líneas de acción que se deben tomar en la estrategia de desarrollo para la actividad pesquera, éstas líneas de acción deben atender las necesidades manifestadas por los productores, tales como construcción de oficinas propias, taller de mantenimiento y almacén de resguardo de bienes, búsqueda de concesión de muelles, renovación de la flota pesquera, crear condiciones para fortalecer el mercado destino del producto y mejora de precios. Para atender algunas de las líneas de acción recomendadas es necesario el financiamiento, el cual se ha visto nulo en el sector pesca.

El estudio aporta pruebas de esfuerzos para desarrollar modelos integrados, donde cada una de las variables micro y macro económicas tiene importancia para la proyección futura de la actividad. Sirve como herramienta para los tomadores de decisiones, así como para la planeación de medidas paliativas y preventivas dirigidas hacia impactos socioeconómicos que se pueden generar en el sector pesquero ante cambios de las condiciones económicas y tecnológicas actuales. 
La metodología es un primer intento de análisis económico y prospectivo de la actividad pesquera y ayuda a sentar las bases para el desarrollo de nueva metodología que pudiera incluir otro tipo de variables.

Agradecimientos: La presente investigación se llevó a cabo dentro de la estancia posdoctoral realizada en CICIMAR-IPN gracias a la beca de CONACYT, AGRADEZCO A AGROPROSPECTA por la capacitación de la metodología MEXSIM y a SAGARPA por las atenciones prestadas durante el proyecto. Finalmente agradezco a los participantes (pescadores) de los paneles de Guaymas, Sonora y Puerto San Carlos, B. C. S. 


\section{Bibliografía}

AGROPROSPECTA (2009), Unidades representativas de Producción, Panorama Económico 2007-2017. Resumen Ejecutivo, Universidad autónoma de Chapingo, Texcoco.

Bernardo, D.J. y Conner J.R. (1990), “Contemporary methodologies in range economics Corvaillis research” (Obemiller, F.W., Ed), Current issues in rangeland resource economics, Oregon State University Extension Service, Oregon, (24-24).

Callejas Juárez, N. (2008), Estudios de caso de los Sistema Producto frutícolas en el Estado de Chihuahua 2007, Universidad Autónoma de Chihuahua, Entidad Evaluadora Estatal y SAGARPA, Chihuahua.

Casas Valdés, M. y G. Ponce Díaz (editores) (1996), Estudio del potencial pesquero y acuícola en Baja California Sur, SEMARNAP, La Paz.

Conner, J.R. (1994), “Assessing the socio-economic impacts of climate change on grazinglands”, (Frederick, K.D. y Rosemberg, N.J., Ed), Climate Change, 143-157.

Hughes, D.W., Richardson, J.W., Rister, M.E. (1985), “Effects of sustained financial stress on the financial structure and performance of the farm sector” American Journal of Agricultural Economics 67, 1116-1122.

Juárez Torres, M., M. de la L. Flores Escobar y J. de Luna Martínez. (2007), El sector pesquero en México, Financiera Rural, Cd. de México.

Knutson, R.D., Romain, R. Anderson, D.P., Richardson, J.W. (1997), “Farmlevel consequences of Canadian and U.S. dairy policies” American Journal of Agricultural Economics 79, 1563-1772.

Knutson, R.D., Smith, E.G., Anderson, D.P., Richardson, J.W. (1998), "Southern farmers exposure to income risk under the 1996 farm bill" Journal of Agricultural and Applied Economics 30, 35-46.

Lien, G. (2003), “Assisting whole-farm decision-making through stochastic budgeting” Agricultiral Systems 76, 399-413.

Nyangito, H.O., Richardson, J.E., Mundy, D.S., Mukhebi, A.W., Zimmel, P., Namkan, J. (1996), "Economic impacts of East Coast Fever immunization on smallholder farms, Kenya: a simulations analysis” Agricultural Economics 13, 163-177.

Ojeda Ruiz de la Peña, M. A. y J. S. Sánchez Hernández. (2003), “Diagnóstico integral del sector pesquero” (Ivanova, A. y M. Ángeles, Ed), Diagnóstico estratégico de Baja California Sur, Universidad Autónoma de Baja California Sur, La Paz, 201-248. 
Quiñonez Velázquez, C, R. Félix Uraga, F. N. Melo Barrera y E. Morales Bojórquez. (2007), "Manejo pesquero: la pesquería de sardina en Baja California Sur” (Urciaga, et al Ed), Recursos marinos y servicios ambientales en el desarrollo regional, Centro de Investigaciones Biológicas del Noroeste, C.S., Universidad Autónoma de Baja California Sur y Centro Interdisciplinario de Ciencias Marinas, La Paz, 197-226.

Richardson, J. W. y Nixon, C.J. (1986), Description of FLIPSIM - V: A general firm level policy simulation model, Texas Agricultural experiment Station Bulletin, College Station, Texas.

SAGARPA (2007), Anuario Estadístico de Acuacultura y Pesca, CONAPESCA, México, D.F.

Souza Neto, J. (1996), Integrating ecological end economic models for assessing institutional, environmental and technological change on rangeland, Department of Rangeland Ecology and Management, Texas A\&M University, College Station, Texas.

Souza Neto, J., Conner, J.R. y Stuth J.W. (2001), “Biophysical and economic models for assessing impacts of change on grazingland ecosystems” Revista Brasileira de Engenharia Agrícola e Ambiental, 135-137.

Recibido: 15.09.2011

Aceptado: 25.07.2012 